\title{
Infinite Horizon Linear Quadratic Gene Regulation in Fluctuating Environments (Technical Report)
}

\author{
Farshad R. Pour Safaei, João P. Hespanha, Stephen R. Proulx
}

\begin{abstract}
We study the evolution of gene regulation in response to environmental fluctuations. For gene dynamics modeled by a linear system, we compute the optimal gene regulator that minimizes the expected square difference between the current protein level and the level that is assumed to be optimal for the current environment plus the cost of protein production/decay, integrated over the life span of the cell. We show that such cost could be represented by a discounted infinite horizon LQR problem with switching equilibria. We also derive a necessary and sufficient condition for the existence of the optimal controller, which can be expressed in terms of a system of Linear Matrix Inequalities (LMI).
\end{abstract}

\section{INTRODUCTION}

Living organisms sense their environmental context and orchestrate the expression of sets of genes to utilize available resources and to survive stressful conditions. Recently, several researchers have considered the effect of stochastically varying environment on gene regulation problems [1], [2], [3]. Following this line of research, we consider a model of gene regulation where the environment switches between discrete states at random time intervals. These states could potentially represent physiological or hormonal states that a cell senses in multicellular organisms. Different environmental conditions have different optimal expression levels, and the performance of the cell improves as the expression level approaches the optimum. For example, a protein that provides a useful function under some environmental conditions may produce deleterious byproducts under other conditions. A recent study of the yeast Saccharomyces cerevisiae found that increasing the expression level of a gene leads to slower growth for one fifth of all genes [4]. Therefore, cells need to adjust their expression level to the level which is optimal for the current environment. Our goal is to consider a cost function that represents the expected cost of deviating from the optimal expression level in the current environment plus the cost of protein production/decay over one individual life span of the cell. We further compute the optimal gene regulation strategy for this problem.

The model that we use to represent the gene regulation problem in fluctuating environments is a special case of

This material is based upon work supported by the National Science Foundation under Grants Nos. ECCS-0725485 and EF-1137835.

F. R. Pour Safaei (farshaddece.ucsb.edu) and J. P. Hespanha (hespanha@ece.ucsb.edu) are with the Dept. of Electrical and Computer Eng., Univ. of California, Santa Barbara, CA 93106; and Stephen R. Proulx (proulxalifesci.ucsb.edu ) is with the Dept. of Ecology, Evolution and Marine Biology, Univ. of California, Santa Barbara, CA 93106, USA.
Piecewise Deterministic Markov (PDM) processes [5] and Stochastic Hybrid Systems (SHS) [6]. SHSs have been frequently used to model gene regulatory networks. For instance, they can be used to model the uncertainties associated with activation/deactivation of a gene in response to the binding/unbinding of proteins to its promoter. By modeling autoregulatory gene networks as a SHS with two discrete states, [7] analyzes the reduction of intrinsic noise caused by the transition of a promoter between its active and inactive states in a genetic network regulated by negative feedback. In [8], this model is extended to a network of $N$ genes. Moreover, SHS models have been shown to be useful for parameter identification and modeling of subtilin production in Bacillus subtilis [9] and nutrient stress response in E. Coli [10].

From the optimal control perspective, several researchers have considered optimization problems on SHSs. [11] studies the Linear Quadratic Regulator problem for Markov Jump Linear (MJL) systems and presents various algorithms to compute the optimal gains. The author of [11] considers both infinite and finite horizon cases and provides a sufficient condition for the existence of solution in the infinite horizon case. Moreover, based on the Stochastic Stabilizability concept for MJL systems, [12] establishes a necessary and sufficient condition for finite cost in the infinite horizon case. Several researchers have constructed iterative algorithms to solve the system of coupled Riccati equations occurring in jump linear control systems. For instance, [13] proposes the construction of a sequence of Lyapunov algebraic equations whose solutions converge to the solution of the coupled Riccati equations that appear in this paper.

Inspired by [14], we model the gene regulation problem in stochastically varying environments in a general framework. We consider linear dynamical models in every environmental condition where the parameters depend on the current environment. We then derive an optimal controller that minimizes a discounted infinite horizon LQR problem with switching equilibria. We also derive a necessary and sufficient condition for the existence of the optimal control, which can be expressed in terms of a system of Linear Matrix Inequalities (LMIs).

When we apply the optimal control results to the computation of optimal gene regulatory responses in variable environments, we conclude that the optimal rate of protein 
production is affine with respect to the current protein level, which turns out to be consistent with the the biologically meaningful model for protein degradation considered in [15]. Our results also show that the optimal control in a variable environment switches between several (affine) feedback laws, one for each environment. However, the feedback law that corresponds to each environment would typically not be optimal for that specific environment, if the environment was static. The implication of this fact is that an organism that evolved toward optimality in a variable environment will generally not be optimal in a static environment that resembles one of the states of its variable environment. Intuitively, this is because the individual will always be trying to anticipate a change that is never realized.

The paper is organized as follows. In Section II, we start by modeling a simple one-step gene expression process with two discrete environments and then generalize it to a $n$-step process with an arbitrary number of environments. In Section III, the optimal control strategy for fluctuating environments is derived, and we establish a necessary and sufficient condition for the existence of solution in terms of LMIs. Section IV provides a case study and we conclude the paper in Section $\mathrm{V}$ with some final conclusions and directions for future research.

Notation. Given a measurable space $(\Omega, \mathfrak{F})$ and probability measure $P: \mathfrak{F} \rightarrow[0,1]$, stochastic process $\mathbf{x}: \Omega \times[0, \infty] \rightarrow$ $\mathcal{X} \subset \mathbb{R}^{n}$ is denoted in boldface.

\section{PROBLEM STATEMENT}

\section{A. Dynamics of a Simple Gene Regulation}

Cells living in complex environments can sense a variety of signals. They monitor their environment through such signals and respond to environmental changes by producing appropriate proteins. The rate of protein productions is determined by transcription regulatory networks composed of genes that code for special proteins called transcription factors [16]. Active transcription factors bind into the promoter region of the DNA and can cause an increase or decrease of the rate at which the target genes are transcribed. The genes are transcribed into mRNA which is then translated into protein. The environmental conditions, mediated through cellular processes, alter the conformation of the transcription factors in a way that affects their binding affinities. It is these changes in the transcription factor proteins that regulate the expression of the target gene, creating positive or negative feedback loops.

We focus on the dynamics of a single gene that is regulated by a single transcription factor. This transcription interaction can be described by $\mathrm{Y} \rightarrow \mathrm{X}$ which reads "transcription factor $Y$ regulates gene $X$ ". Once the transcription factor $Y$ activates the gene $X$, it begins to be transcribed, the mRNA is translated, and this results in the accumulation of protein $\mathrm{X}$. We assume that the rate of protein production is denoted by $\mathbf{u}$ (in units of concentration per unit of time).
The process of protein production is balanced by two additional processes: protein degradation (protein destruction by specialized proteins in the cell) and dilution (due to increase of the cell volume during growth). We denote the total degradation/dilution rate by $\mu$ which is the sum of the degradation rate $\mu_{d e g}$ and the dilution rate $\mu_{d i l}$. Thus, the change of concentration of $\mathrm{X}$ can be described by the dynamic equation

$$
\frac{d \mathbf{x}}{d t}=\mathbf{u}-\mu \mathbf{x}
$$

where $\mathbf{x}$ describes the protein concentration.

\section{B. Gene Regulation in Fluctuating Environments}

We consider a cell encountering a series of environmental conditions and our goal is to understand what the optimal gene regulation strategy is.

Let us start by assuming that the cell encounters two different environmental conditions: environment 0 favors low concentration of protein while environment 1 favors high concentration. These conditions may represent physical parameters such as temperature or osmotic pressure, signaling molecules from other cells, beneficial nutrients, or harmful chemicals. The random environmental shifts are modeled by exponential waiting times with parameters $\lambda_{i}$, for which the history does not influence the future states. Given this definition, the expected waiting that the environment stays in state $i$ is $\frac{1}{\lambda_{1-i}}$ for $i \in\{0,1\}$.

We start by considering a scenario where the optimal concentration of the protein $\mathrm{X}$ depends on the current environment, denoted by $\operatorname{env}(t) \in\{0,1\}$, and the degradation rate is constant. The evolution of protein concentration $\mathbf{x}(t)$ can be modelled by

$$
\frac{d \mathbf{x}}{d t}=\mathbf{u}_{\mathrm{env}}-\mu \mathbf{x}(t)
$$

where $\mathbf{u}_{i}$ is the rate of transcription in environment $i \in\{0,1\}$ and $\mu$ is the protein degradation/dilution rate. Figure 1 shows a sample path of the resulting stochastic system due to changing environments.

Let us consider a simple evolutionary scenario. We assume that the optimal concentration levels of the protein $\mathrm{X}$ are 0 and 1 in environments 0 and 1, respectively. At each point in time, we assume that cost of deviation of the protein level from the optimal level in the current environment is a quadratic function of the difference between these values. This cost can be written as $(\mathbf{x}(t)-\mathbf{e n v}(t))^{2}$, since we assumed that the optimal protein levels are 0 and 1 in environments 0 and 1 , respectively.

We also consider a term in the cost function that reflects energetic costs of producing/decaying mRNA and proteins [17]. This cost may be written as a quadratic function of the current transcription rate $\mathbf{u}(t)$, resulting in a total cost that is given by $(\mathbf{x}-\mathbf{e n v})^{2}+\gamma \mathbf{u}^{2}$ and defines the penalty in environment env associated with the protein concentration $\mathbf{x}$ plus the cost of instantaneous protein production/decay. The parameter $\gamma$ determines the tradeoff between keeping $\mathbf{x}(t)$ 


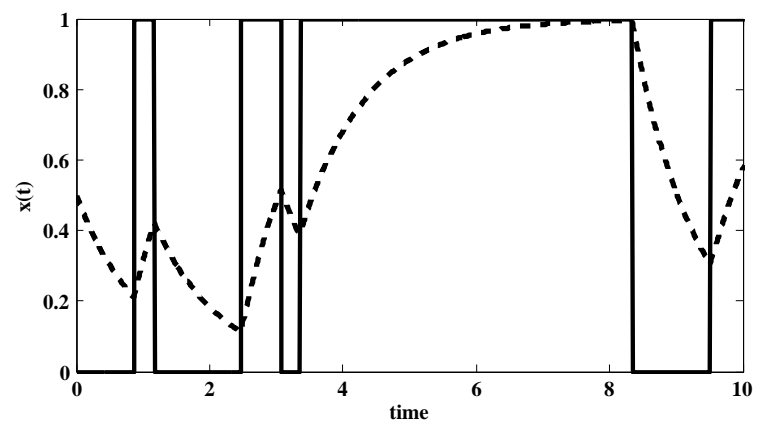

Fig. 1. A sample path over one individual's life span. The solid line illustrates how the environment changes stochastically while the trajectory of the protein concentration $\mathbf{x}(t)$ over one sample path is depicted by the dashed line.

close to its ideal value env $(t)$ and not "wasting" resources in the protein production/decay. One can also consider the case in which $\gamma$ is environment-dependent, which will be discussed in the following subsection.

We assume that organisms die at a rate independent of the strategy they use to regulate gene expression. If the life span $(\mathbf{T})$ of a cell is modeled by an exponential random variable with mean $1 / \rho$, the probability that an organism is still alive at age $t$ is given by $P(\mathbf{T}>t)=e^{-\rho t}$. This assumption is consistent with the experimental data in [18], [19] and [20]. One can show that the total expected lifetime cost of an individual is proportional to

$$
\mathbf{J}=\int_{0}^{\infty} e^{-\rho t}\left((\mathbf{x}(t)-\mathbf{e n v}(t))^{2}+\gamma \mathbf{u}(t)^{2}\right) d t .
$$

Equation (2) provides the cost associated with a specific realization of the stochastic process env $(t)$ that models environmental changes. Since an individual cannot "guess" the future evolution of env $(t)$, its best bet is to minimize the expected value of such cost, given the current environment and concentration of $\mathbf{x}$

$$
J=E_{z_{0}}\left\{\int_{0}^{\infty} e^{-\rho t}\left((\mathbf{x}(t)-\mathbf{e n v}(t))^{2}+\gamma \mathbf{u}(t)^{2}\right) d t\right\}
$$

conditioned upon the initial condition $z_{0}=(\mathbf{x}(0), \operatorname{env}(0))$.

One can also interpret (2) by considering a "killed process" $\overline{\mathrm{x}}$ that is equal to $\mathrm{x}$ as long as the cell is alive and $\overline{\mathrm{x}}=\mathbf{e n v}$ after the organism is dead (which generated no further cost with the control $\mathbf{u}=0$ ), the total lifetime cost of the killed process is

$$
\tilde{J}=E_{z_{0}}\left\{\int_{0}^{\infty}(\overline{\mathbf{x}}(t)-\operatorname{env}(t))^{2}+\gamma \mathbf{u}(t)^{2} d t\right\} .
$$

It can be shown that the killed process generates the same cost as (3), i.e. $\tilde{J}=J$, see [5, Chapter 3].

\section{Generalization}

We now generalize the system described above by considering a multiple-step gene expression process with an arbitrary number of environmental conditions. This can be used to model the multiple-step process in gene production (e.g., the transcription-translation process DNA $\rightarrow$ mRNA $\rightarrow$ protein) and also regulation based on multiple transcription factors.

We model the process of switching between environments by a continuous-time Markov chain $\mathbf{r}(t)$ taking values in the set $\mathcal{S}=\{1,2, \ldots, N\}$ with transition rate matrix $\mathbf{P}:=\left\{\lambda_{i j}\right\}$ where

$$
P(\mathbf{r}(t+d t)=j \mid \mathbf{r}(t)=i)=\lambda_{i j} d t+O(d t) \quad i \neq j .
$$

Here, $\lambda_{i j} \geq 0(i \neq j)$ is the rate of departing from state $i$ to state $j$ and $\lambda_{i i}=-\sum_{j=1, j \neq i}^{N} \lambda_{i j}$.

The different values of $\mathbf{r}(t)$ correspond to distinct linear dynamics according to the following model:

$$
\dot{\mathbf{x}}(t)=A_{\mathbf{r}(t)} \mathbf{x}(t)+B_{\mathbf{r}(t)} \mathbf{u}(t)+d_{\mathbf{r}(t)}
$$

where $\mathbf{x}(t) \in \mathbb{R}^{n}$ denotes a stochastic process state with piecewise continuous sample paths, $\mathbf{r}(t)$ denotes the current environmental condition, $\mathbf{u}(t) \in \mathbb{R}^{m}$ an input to be optimized, and $d_{\mathbf{r}(t)}$ is an $\mathbf{r}$-dependent bias term. The affine term $d_{\mathbf{r}(t)}$ in the dynamics is needed for environments that create or consume $\mathrm{x}$ at a fixed rate without control cost.

\section{MAIN RESULTS}

Our goal is to compute the optimal control input $\mathbf{u}(t)$ that minimizes an infinite-horizon discounted criteria of the following form

$$
\begin{aligned}
J=E_{z_{0}}\left\{\int_{0}^{\infty}\right. & e^{-\rho t}\left(\left(\mathbf{x}-\bar{x}_{\mathbf{r}}\right)^{\prime} Q_{\mathbf{r}}\left(\mathbf{x}-\bar{x}_{\mathbf{r}}\right)\right. \\
& \left.\left.+\left(\mathbf{u}-\bar{u}_{\mathbf{r}}\right)^{\prime} R_{\mathbf{r}}\left(\mathbf{u}-\bar{u}_{\mathbf{r}}\right)\right) d t\right\}
\end{aligned}
$$

by means of a feedback policy that computes $\mathbf{u}(t)$ based on the history of $\mathbf{x}(\tau)$ and $\mathbf{r}(\tau), \forall \tau \leq t$ where all the $Q_{i}$ and $R_{i}$ are positive definite matrices. Toward this goal, we shall provide a necessary and sufficient condition for the existence of solution, which requires the notion of stochastic stabilizability that we have adapted from [12].

Consider a Markov Jump Linear (MJL) system given by (4)-(5) and let $\mathbf{x}\left(t, x_{0}, r_{0}, \mathbf{u}\right)$ denote the trajectory of the process starting from initial condition $z_{0}=\left(\mathbf{x}\left(t_{0}\right), \mathbf{r}\left(t_{0}\right)\right)=$ $\left(x_{0}, r_{0}\right)$, and under the control input $\mathbf{u}(t)$. The system is Stochastically Stabilizable (SS) if there exist a symmetric matrix $M$ and a set of linear gains $\left\{L_{i}: i \in \mathcal{S}\right\}$ such that the solution of (4)-(5) with $d_{i}=0$ and $\mathbf{u}(t)=-L_{\mathbf{r}(t)} \mathbf{x}(t)$ satisfies

$$
\lim _{T \rightarrow \infty} E_{z_{0}}\left\{\int_{0}^{T} \mathbf{x}(t)^{\prime} \mathbf{x}(t) d t\right\} \leq x_{0}^{\prime} M x_{0}
$$

for all finite $x_{0} \in \mathbb{R}^{n}$ and $r_{0} \in \mathcal{S}$. Essentially, stochastic stabilizability of a system is equivalent to the existence of a set of linear feedback gains that make the state mean-square integrable when $d_{i}=0 \forall i \in \mathcal{S}$. The next result from [12, Theorem 1] provides a necessary and sufficient condition for stochastic stabilizability of MJL systems. 
Theorem 3.1: The system (4)-(5) is stochastically stabilizable if and only if there exists a set of matrices $\left\{L_{i}: i \in \mathcal{S}\right\}$ such that for every set of positive definite symmetric matrices $\left\{N_{i}: i \in \mathcal{S}\right\}$, the symmetric solutions $\left\{M_{i}: i \in \mathcal{S}\right\}$ of the coupled equations

$$
\left(A_{i}-B_{i} L_{i}\right)^{\prime} M_{i}+M_{i}\left(A_{i}-B_{i} L_{i}\right)+\sum_{j=1}^{N} \lambda_{i j} M_{j}=-N_{i}
$$

are positive definite for all $i \in \mathcal{S}$.

In the following theorem, we compute the optimal control policy $\mathbf{u}^{*}(t)$ that minimizes the infinite-horizon discounted criteria (6). Such control signal may depend on the values of $\mathbf{x}(\tau)$ and $\mathbf{r}(\tau)$ for $\tau \leq t$.

Theorem 3.2: Consider the following optimization problem

$$
\min _{u(\mathbf{x}(\tau), \mathbf{r}(\tau)), \tau \leq t} J
$$

subject to $\quad \dot{\mathbf{x}}(t)=A_{\mathbf{r}(t)} \mathbf{x}(t)+B_{\mathbf{r}(t)} \mathbf{u}(t)+d_{\mathbf{r}(t)}$

with $J$ given by (6). If there exists a solution $\Lambda_{i} \in \mathbb{R}^{n \times n}$, $\Gamma_{i} \in \mathbb{R}^{n}, \Omega_{i} \in \mathbb{R}, i \in \mathcal{S}$ to the following set of equations

$$
\begin{aligned}
& A_{i}^{\prime} \Lambda_{i}+\Lambda_{i} A_{i}-\rho \Lambda_{i}-\Lambda_{i} B_{i} R_{i}^{-1} B_{i}^{\prime} \Lambda_{i}+Q_{i}+\sum_{j=1}^{N} \lambda_{i j} \Lambda_{j}=0 \\
& \quad\left(A_{i}^{\prime}-\Lambda_{i} B_{i} R_{i}^{-1} B_{i}^{\prime}-\rho I\right) \Gamma_{i}+2 \Lambda_{i}\left(B_{i} \bar{u}_{i}+d_{i}\right) \\
& +\sum_{j=1}^{N} \lambda_{i j} \Gamma_{j}=2 Q_{i} \bar{x}_{i} \\
& \quad-\frac{1}{4} \Gamma_{i}^{\prime} B_{i} R_{i}^{-1} B_{i}^{\prime} \Gamma_{i}+\Gamma_{i}^{\prime}\left(B_{i} \bar{u}_{i}+d_{i}\right)-\rho \Omega_{i} \\
& \quad+\sum_{j=1}^{N} \lambda_{i j} \Omega_{j}+\bar{x}_{i}^{\prime} Q_{i} \bar{x}_{i}=0
\end{aligned}
$$

then the minimal cost for $\mathbf{x}(0)=x_{0}, \mathbf{r}(0)=r_{0}$ is given by $J^{*}=x_{0}^{\prime} \Lambda_{r_{0}} x_{0}+x_{0}^{\prime} \Gamma_{r_{0}}+\Omega_{r_{0}}$ and the optimal control is given by

$$
\mathbf{u}^{*}(t)=\bar{u}_{\mathbf{r}}-\frac{1}{2} R_{\mathbf{r}}^{-1} B_{\mathbf{r}}^{\prime}\left(2 \Lambda_{\mathbf{r}} \mathbf{x}+\Gamma_{\mathbf{r}}\right) \quad \mathbf{r}:[0, \infty) \rightarrow \mathcal{S} .
$$

Proof of Theorem 3.2: Let us introduce the value function as $V\left(x_{0}, r_{0}\right)=\min _{u} J$ conditioned on $\mathbf{x}(0)=$ $x_{0}, \mathbf{r}\left(t_{0}\right)=r_{0}$. From [5], the Hamilton-Jacobi-Bellman (HJB) equation for this problem is given by

$$
\begin{aligned}
0= & \min _{u}\left\{\mathcal{L} V(x, i)-\rho V(x, i)+\left(x-\bar{x}_{i}\right)^{\prime} Q_{i}\left(x-\bar{x}_{i}\right)\right. \\
& \left.+\left(u-\bar{u}_{i}\right)^{\prime} R_{i}\left(u-\bar{u}_{i}\right)\right\}
\end{aligned}
$$

where $\mathcal{L} V$ denotes the extended generator of the Markov pair $\{\mathbf{r}(t), \mathbf{x}(t)\}$, see [6]. The minimization in (14) can be done explicitly, leading to the optimal feedback

$$
u^{*}=\bar{u}_{i}-\frac{1}{2} R_{i}^{-1} B_{i}^{\prime}\left(\frac{\partial V}{\partial x}\right)^{\prime},
$$

that can be replaced in (14). Using (10)-(12), it is straightforward to verify that $V(x, i)=x^{\prime} \Lambda_{i} x+x^{\prime} \Gamma_{i}+\Omega_{i}$ is a piecewise continuous solution to (14), since

$$
\begin{aligned}
0= & \frac{\partial V}{\partial x}\left(A_{i} x+B_{i} u^{*}+d_{i}\right)+\sum_{j=1}^{N} \lambda_{i j}\left(x^{\prime} \Lambda_{j} x+x^{\prime} \Gamma_{j}+\Omega_{j}\right) \\
& -\rho\left(x^{\prime} \Lambda_{i} x+x^{\prime} \Gamma_{i}+\Omega_{i}\right)+\left(x-\bar{x}_{i}\right)^{\prime} Q_{i}\left(x-\bar{x}_{i}\right) \\
& +\left(u^{*}-\bar{u}_{i}\right)^{\prime} R_{i}\left(u^{*}-\bar{u}_{i}\right) .
\end{aligned}
$$

Thus, by [5, 42.8], $V$ and $\mathbf{u}^{*}$ are optimal which completes the proof.

Next, a necessary and sufficient condition for the existence of the optimal regulator will be stated in terms of stochastic stabilizability of the system. We show that under a stochastic stabilizability assumption, the optimal control policy leads to a finite cost for which one can compute a finite upper bound on $J$. The main result of this section is stated in the following theorem.

Theorem 3.3: Consider the system (4)-(5) and (6) and assume that $\rho>-\lambda_{i i}$ for all $i \in \mathcal{S}$. When the system is stochastically stabilizable, the minimum cost is finite, the equations (10)-(12) have solutions, and the control policy (13) is optimal. Conversely, if for some linear policy the cost (6) is bounded then the system is stochastically stabilizable.

Proof of Theorem 3.3: We start by proving the first part of the theorem by showing that Stochastic Stabilizability results in a finite optimal cost. Then, we show that there exists a solution to (10)-(12) and therefore the optimality of (13) follows from Theorem 3.2.

Due to the stochastic stabilizability assumption (Theorem 3.1), there exists a set of gains $\left\{L_{i}\right\}$ such that for any set of matrices $\left\{\tilde{N}_{i}>0\right\}$, the corresponding solutions $\left\{\tilde{M}_{i}\right\}$ in (8) are positive definite. In what follows, we show that choosing the control $u(t)=-L_{\mathbf{r}} \mathbf{x}(t)$ (which is not necessarily optimal) results in a finite cost.

We take matrices $N_{i}$ in (8) to be $N_{i}=Q_{i}+L_{i}^{\prime} R_{i} L_{i}>0$ and $M_{i}$ to be the corresponding positive definite solutions. Given $\mathbf{x}(0)=x_{0}$ and $\mathbf{r}(0)=r_{0}$, one can compute the cost of applying this control policy using

$$
\begin{aligned}
J & =E_{z_{0}}\left\{\int _ { 0 } ^ { \infty } e ^ { - \rho t } \left(\left(\mathbf{x}-\bar{x}_{\mathbf{r}}\right)^{\prime} Q_{\mathbf{r}}\left(\mathbf{x}-\bar{x}_{\mathbf{r}}\right)\right.\right. \\
& \left.\left.+\left(u-\bar{u}_{\mathbf{r}}\right)^{\prime} R_{\mathbf{r}}\left(u-\bar{u}_{\mathbf{r}}\right)\right) d t\right\} \\
& =E_{z_{0}}\left\{\int _ { 0 } ^ { \infty } e ^ { - \rho t } \left(\mathbf{x}^{\prime} N_{\mathbf{r}} \mathbf{x}-2 \mathbf{x}^{\prime}\left(Q_{\mathbf{r}} \bar{x}_{\mathbf{r}}+L_{\mathbf{r}}^{\prime} R_{\mathbf{r}} \bar{u}_{\mathbf{r}}\right)\right.\right. \\
& \left.\left.+\bar{u}_{\mathbf{r}}^{\prime} R_{\mathbf{r}} \bar{u}_{\mathbf{r}}+\bar{x}_{\mathbf{r}}^{\prime} Q_{\mathbf{r}} \bar{x}_{\mathbf{r}}\right) d t\right\} .
\end{aligned}
$$

Defining $W(\mathbf{x}, \mathbf{r})=\mathbf{x}^{\prime} M_{\mathbf{r}} \mathbf{x}$ and applying the extended generator of the stochastic system (5)-(4), see [6], we obtain $\mathcal{L} W(\mathbf{x}, \mathbf{r})=-\mathbf{x}^{\prime} N_{\mathbf{r}} \mathbf{x}$. So one can show that

$$
\frac{\mathcal{L} W}{W}=-\frac{\mathbf{x}^{\prime} N_{\mathbf{r}} \mathbf{x}}{\mathbf{x}^{\prime} M_{\mathbf{r}} \mathbf{x}} \leq-\alpha \quad \alpha:=\min _{i} \frac{\mu_{\min }\left(N_{i}\right)}{\mu_{\max }\left(M_{i}\right)} \quad i \in \mathcal{S}
$$

where $\alpha$ is positive. So $\mathcal{L} W \leq-\alpha W$ and by the GronwallBellman lemma

$$
E_{z_{0}}\{W(\mathbf{x}, \mathbf{r})\} \leq e^{-\alpha t} W\left(x_{0}, r_{0}\right) .
$$




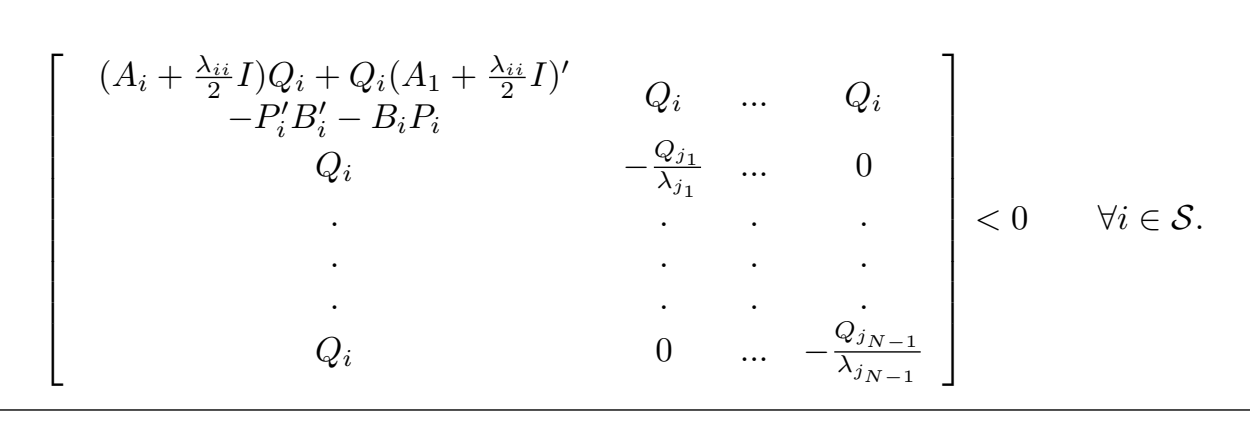

Thus, one can conclude that

$$
E_{z_{0}}\left\{\int_{0}^{T} \mathbf{x}^{\prime} M_{\mathbf{r}} \mathbf{x} d t\right\} \leq\left(\int_{0}^{T} e^{-\alpha t} d t\right) x_{0}^{\prime} M_{r_{0}} x_{0} .
$$

Lebesgue's Dominated Convergence Theorem in [21] justifies the existence of the limit as $T \rightarrow \infty$ and we have

$$
\begin{aligned}
E_{z_{0}}\left\{\int_{0}^{\infty} \mathbf{x}^{\prime} M_{\mathbf{r}} \mathbf{x} d t\right\} & =\lim _{T \rightarrow \infty} E_{z_{0}}\left\{\int_{0}^{T} \mathbf{x}^{\prime} M_{\mathbf{r}} \mathbf{x} d t\right\} \\
& \leq \frac{1}{\alpha} x_{0}^{\prime} M_{r_{0}} x_{0}
\end{aligned}
$$

We can bound the integral (17) which can be written as

$$
\begin{aligned}
J & =E_{z_{0}}\left\{\int_{0}^{\infty} e^{-\rho t}\left(\bar{u}_{\mathbf{r}}^{\prime} R_{\mathbf{r}} \bar{u}_{\mathbf{r}}+\bar{x}_{\mathbf{r}}^{\prime} Q_{\mathbf{r}} \bar{x}_{\mathbf{r}}\right) d t\right\} \\
& +E_{z_{0}}\left\{\int_{0}^{\infty} e^{-\rho t}\left(\mathbf{x}^{\prime} N_{\mathbf{r}} \mathbf{x}\right) d t\right\} \\
& -2 E_{z_{0}}\left\{\int_{0}^{\infty} e^{-\rho t} \mathbf{x}^{\prime}\left(Q_{\mathbf{r}} \bar{x}_{\mathbf{r}}+L_{\mathbf{r}}^{\prime} R_{\mathbf{r}} \bar{u}_{\mathbf{r}}\right) d t\right\}
\end{aligned}
$$

Since $\mathcal{S}$ is finite, the first term in (19) can be bounded by

$$
\begin{aligned}
E_{z_{0}} & \left\{\int_{0}^{\infty} e^{-\rho t}\left(\bar{u}_{\mathbf{r}}^{\prime} R_{\mathbf{r}} \bar{u}_{\mathbf{r}}+\bar{x}_{\mathbf{r}}^{\prime} Q_{\mathbf{r}} \bar{x}_{\mathbf{r}}\right) d t\right\} \\
& \leq \frac{1}{\rho} \max _{i \in \mathcal{S}}\left(\bar{u}_{i}^{\prime} R_{i} \bar{u}_{i}+\bar{x}_{i}^{\prime} Q_{i} \bar{x}_{i}\right) .
\end{aligned}
$$

For the second integral in (19), we have

$$
\begin{aligned}
E_{z_{0}}\left\{\int_{0}^{\infty}\right. & \left.e^{-\rho t} \mathbf{x}^{\prime} N_{\mathbf{r}} \mathbf{x} d t\right\} \\
& \leq \frac{\max _{i} \mu_{\max }\left(N_{i}\right)}{\min _{i} \mu_{\min }\left(M_{i}\right)} E_{z_{0}}\left\{\int_{0}^{\infty} e^{-\rho t} \mathbf{x}^{\prime} M_{\mathbf{r}} \mathbf{x} d t\right\} \\
& \leq \frac{\max _{i} \mu_{\max }\left(N_{i}\right)}{\min _{i} \mu_{\min }\left(M_{i}\right)} \cdot \frac{1}{\alpha} x_{0}^{\prime} M_{r_{0}} x_{0}
\end{aligned}
$$

and the third one can be bounded by

$$
\begin{aligned}
& E_{z_{0}}\left\{\int_{0}^{\infty} e^{-\rho t} \mathbf{x}^{\prime}\left(Q_{\mathbf{r}} \bar{x}_{\mathbf{r}}+L_{\mathbf{r}}^{\prime} R_{\mathbf{r}} \bar{u}_{\mathbf{r}}\right) d t\right\} \leq \\
& \max _{i}\left|Q_{i} \bar{x}_{i}+L_{i}^{\prime} R_{i} \bar{u}_{i}\right| E_{z_{0}}\left\{\int_{0}^{\infty} e^{-\rho t}|\mathbf{x}| d t\right\}
\end{aligned}
$$

Defining $\kappa:=\max \left|Q_{i} \bar{x}_{i}+L_{i}^{\prime} R_{i} \bar{u}_{i}\right|$, and using the Cauchy Schwarz inequality for square integrable functions

$$
\begin{aligned}
\kappa E_{z_{0}}\left\{\int_{0}^{\infty}\right. & \left.e^{-\rho t}|\mathbf{x}| d t\right\} \\
& \leq \kappa E_{z_{0}}\left\{\sqrt{\int_{0}^{\infty} e^{-2 \rho t} d t \int_{0}^{\infty}|\mathbf{x}|^{2} d t}\right\} \\
& =\frac{\kappa}{\sqrt{2 \rho}} E_{z_{0}}\left\{\sqrt{\int_{0}^{\infty}|\mathbf{x}|^{2} d t}\right\} \\
& \leq \frac{\kappa}{\sqrt{2 \rho \min _{i} \mu_{\min }\left(M_{i}\right)}} E_{z_{0}}\left\{\sqrt{\int_{0}^{\infty} \mathbf{x}^{\prime} M_{\mathbf{r}} \mathbf{x} d t}\right\}
\end{aligned}
$$

Note that, by the Cauchy Schwarz inequality, one can show that $E\{X\} \leq \sqrt{E\left\{X^{2}\right\}}$, so

$$
\begin{aligned}
& \frac{\kappa}{\sqrt{2 \rho \min _{i} \mu_{\min }\left(M_{i}\right)}} E_{z_{0}}\left\{\sqrt{\int_{0}^{\infty} \mathbf{x}^{\prime} M_{\mathbf{r}} \mathbf{x} d t}\right\} \\
& \leq \frac{\kappa}{\sqrt{2 \rho \min _{i} \mu_{\min }\left(M_{i}\right)}} \sqrt{E_{z_{0}}\left\{\int_{0}^{\infty} \mathbf{x}^{\prime} M_{\mathbf{r}} \mathbf{x} d t\right\}} \\
& \leq \frac{\kappa}{\sqrt{2 \rho \min _{i} \mu_{\min }\left(M_{i}\right)}} \cdot \sqrt{\frac{1}{\alpha} x_{0}^{\prime} M_{r_{0}} x_{0}},
\end{aligned}
$$

therefore the cost is bounded. This finite quantity (resulting from a not necessarily optimal control) is an upper bound for the optimal cost to go.

We now show that (10)-(12) has a solution and therefore the optimality of (13) follows from Theorem 3.2. Due to the Stochastic Stabilizability assumption, one can guarantee the existence of a set of positive solutions $\Lambda_{i}$ to (10) [12]. From (10), it is straightforward to show that $A_{i}-B_{i} R_{i}^{-1} B_{i}^{\prime} \Lambda_{i}+$ $\left(\lambda_{i i}-\rho\right) / 2 I$ is Hurwitz. Let us define

$k:=\min \left|\operatorname{Real}\left\{\operatorname{eig}\left(A_{i}-B_{i} R_{i}^{-1} B_{i}^{\prime} \Lambda_{i}+\left(\lambda_{i i}-\rho\right) / 2 I\right)\right\}\right|$ therefore $\left(A_{i}-B_{i} R_{i}^{-1} B_{i}^{\prime} \Lambda_{i}+\left(\lambda_{i i}-\rho+k\right) / 2 I\right)$ is Hurwitz. Since, by assumption $\rho>-\lambda_{i i}$, one can conclude that $\left(A_{i}-B_{i} R_{i}^{-1} B_{i}^{\prime} \Lambda_{i}+(k / 2-\rho) I\right)$ is a stable matrix. Moreover, knowing $\Lambda_{i}$, (11) turns out to be a system of linear equations in $\Gamma_{i}$. Stacking all the entries of the matrix $\Gamma_{i}$ in a tall column vector $z \in \mathbb{R}^{n^{2}}$, we can write (11) as $M z=w$ for an appropriately defined vector $w \in \mathbb{R}^{n^{2}}$ and with the coefficient matrix $M$ defined as

$M=\left(\mathbf{P}-\frac{k}{2} I\right) \otimes I_{n}+\operatorname{diag}\left(A_{i}^{\prime}-\Lambda_{i} B_{i} R_{i}^{-1} B_{i}^{\prime}+\left(\frac{k}{2}-\rho\right) I\right)$.

By the results of [23], the eigenvalues of the transition rate matrix $\mathbf{P}$ are zero or negative therefore $\left(\mathbf{P}-\frac{k}{2} I\right) \otimes I_{n}$ is also Hurwitz. Thus, the system of linear equations (11) has a full rank coefficient matrix and has a unique solution. Similarly, knowing the solution of (10)-(11), (12) turns out to be a system of linear equations in $\Omega_{i}$ with the coefficient matrix $\mathbf{P}-\rho I$. Since all the eigenvalues of $\mathbf{P}-\rho I$ are negative, the coefficient matrix is full rank and (12) has a unique solution.

To prove the second part of the Theorem, suppose that the system is not stochastically stabilizable. So there is no linear feedback law that can result in a finite value for (7), and this contradicts the existence of a finite cost for a linear policy. 
Theorem 3.3 provides a necessary and sufficient condition for the existence of the optimal solution in terms of stochastic stabilizability property. However, for a given set of matrices $\left\{N_{i}\right\}$, the matrix equality (8) is bilinear in the unknowns $\left\{L_{i}\right\},\left\{M_{i}\right\}$ and therefore it is not easy to verify if it holds. The following result provides a system of linear matrix inequalities (LMIs) that can be equivalently used to check stochastic stabilizability. Checking feasibility of these LMIs corresponds to a convex optimization problem that can be solved efficiently.

Lemma 3.1: The following statements are equivalent.

A) The system (4)-(5) is stochastically stabilizable.

B) There exist sets of matrices $\left\{L_{i}\right\}$ and $\left\{M_{i}=M_{i}^{\prime}>\right.$ $0\}$ such that the following Bilinear Matrix Inequality (BMI) holds

$$
\left(A_{i}-B_{i} L_{i}\right)^{\prime} M_{i}+M_{i}\left(A_{i}-B_{i} L_{i}\right)+\sum_{j=1}^{N} \lambda_{i j} M_{j}<0 .
$$

C) There exist sets of matrices $\left\{P_{i}\right\}$ and $\left\{Q_{i}=Q_{i}^{\prime}>0\right\}$ such that the LMI condition (18) holds for $\forall i \in \mathcal{S}$ and $j_{k} \in \mathcal{S} \backslash\{i\}$.

Moreover, the matrices in (B) and (C) are related by $Q_{i}=$ $M_{i}^{-1}$ and $P_{i}=L_{i} Q_{i}$.

Proof of Lemma 3.1: We start by showing that (A) and (B) are equivalent. If the system is stochastically stabilizable, it follows from Theorem 3.1 that there exist matrices $\left\{L_{i}\right\}$ such that for any set of positive definite matrices $\left\{N_{i}\right\}$, the solution $\left\{M_{i}\right\}$ to (8) are positive definite. By selecting $\left\{N_{i}=I\right\}$ in (8), we conclude that (20) holds, which proves that stochastic stabilizability is a sufficient condition for (20) to hold. To prove necessity, let us assume that the $\left\{L_{i}\right\}$, $\left\{M_{i}\right\}$ are such that for some $\left\{N_{i}\right\}$ we have $\left(A_{i}-B_{i} L_{i}\right)^{\prime} M_{i}+$ $M_{i}\left(A_{i}-B_{i} L_{i}\right)+\sum_{j=1}^{N} \lambda_{i j} M_{j}=-N_{i}<0$. Our goal is to show that the system is stochastically stabilizable. Let $V(\mathbf{x}, \mathbf{r})=\mathbf{x}^{\prime} M_{\mathbf{r}} \mathbf{x}$ be the stochastic Lyapunov function for the system where $\left\{M_{i}: i \in \mathcal{S}\right\}$ satisfy (20). Applying the results in [6] to the generator of stochastic hybrid systems, one can compute the time derivative of the expected value of $V$ along the solutions of (4)-(5). Given any $\mathbf{x}(0)=$ $x_{0}, \mathbf{r}(0)=r_{0}$,

$$
\begin{aligned}
\frac{d}{d t} E_{z_{0}}\{V(\mathbf{x}, \mathbf{r})\} \quad & =E_{z_{0}}\left\{\mathbf { x } ^ { \prime } \left(M_{\mathbf{r}}\left(A_{\mathbf{r}}-B_{\mathbf{r}} L_{\mathbf{r}}\right)+\right.\right. \\
& \left.\left.\left(A_{\mathbf{r}}-B_{\mathbf{r}} L_{\mathbf{r}}\right)^{\prime} M_{\mathbf{r}}+\sum_{j=1}^{N} \lambda_{\mathbf{r} j} M_{j}\right) \mathbf{x}\right\}
\end{aligned}
$$

Let us define $\alpha:=\min _{i \in \mathcal{S}} \frac{\lambda_{\min }\left(N_{i}\right)}{\lambda_{\max }\left(M_{i}\right)}$ which is a positive number therefore

$$
\frac{d}{d t} E_{z_{0}}\{V(\mathbf{x}, \mathbf{r})\} \leq-\alpha E_{z_{0}}\{V(\mathbf{x}, \mathbf{r})\} .
$$

Using the Gronwall-Bellman lemma

$$
E_{z_{0}}\{V(\mathbf{x}, \mathbf{r})\} \leq e^{-\alpha t} x_{0}^{\prime} M_{r_{0}} x_{0} .
$$

Thus one can conclude

$$
E_{z_{0}}\left\{\int_{0}^{T} \mathbf{x}(t)^{\prime} M_{\mathbf{r}} \mathbf{x}(t) d t\right\} \leq\left(\int_{0}^{T} e^{-\alpha t} d t\right) x_{0}^{\prime} M_{r_{0}} x_{0} .
$$

Lebesgue's Dominated Convergence Theorem in [21] justifies the existence of the limit as $T \rightarrow \infty$ and we have

$$
\lim _{T \rightarrow \infty} E_{z_{0}}\left\{\int_{0}^{T} \mathbf{x}(t)^{\prime} M_{\mathbf{r}} \mathbf{x}(t) d t\right\} \leq x_{0}^{\prime}\left(\max _{i} \frac{M_{i}}{\alpha\left\|M_{i}\right\|}\right) x_{0} .
$$

Therefore, the system is stochastically stabilizable.

We now prove that (B) and (C) are also equivalent. We sketch the proof for $\mathcal{S}=\{1,2,3\}$ although similar results hold for arbitrarily number of modes. Assume that there exist matrices $\left\{M_{i}\right\}$ and $\left\{L_{i}\right\}$ such that

$$
\left(A_{i}-B_{i} L_{i}\right)^{\prime} M_{i}+M_{i}\left(A_{i}-B_{i} L_{i}\right)+\sum_{j=1}^{3} \lambda_{i j} M_{i}<0 .
$$

Define $Q_{i}:=M_{i}^{-1}>0$ and $P_{i}:=L_{i} Q_{i}$, and multiply both sides of (21) by $Q_{i}$

$$
\begin{aligned}
& \left(A_{i}+\frac{\lambda_{i i}}{2} I\right) Q_{i}+Q_{i}\left(A_{i}+\frac{\lambda_{i i}}{2} I\right)^{\prime}-P_{i}^{\prime} B_{i}^{\prime}-B_{i} P_{i}+ \\
& \lambda_{i j_{1}} Q_{i} Q_{j_{1}}^{-1} Q_{i}+\lambda_{i j_{2}} Q_{i} Q_{j_{2}}^{-1} Q_{1}<0 .
\end{aligned}
$$

Applying the Schur complement [22] to (22), one can get

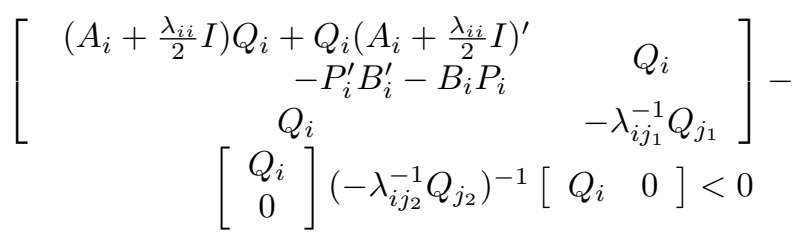

for $\forall i \in \mathcal{S}, j_{k} \in \mathcal{S} \backslash\{i\}$. By applying the Schur complement again, we get (18). Moreover, the proof of necessity follows in a similar fashion. Therefore (B) and (C) are actually equivalent, and this completes the proof.

\section{CASE STUDY}

We consider again the simple gene regulation problem from Section II-B. It can be shown that the system (1) is stochastically stabilizable for any set of parameters $\left\{\lambda_{0}, \lambda_{1}, \mu\right\}$ and, using Theorem 3.2, one can compute the optimal control (13) for this stochastic process.

Let us consider two different scenarios. First, we consider the optimal control policy (13) that is obtained for the stochastically varying environment. Second, we compute two policies that are optimal for environments 0 and 1 individually, assuming that there is no fluctuation in the environment. These policies are obtained by minimizing the cost (2) when the probability of changing the environment is zero. If cells were to use these policies when the environment fluctuates, one can show that the cost of applying this control is a quadratic function of the initial protein concentration and depends on the initial environment. Clearly, such cost is always larger than the optimal cost obtained from Theorem 3.2. 
Fig. a: Optimal cost

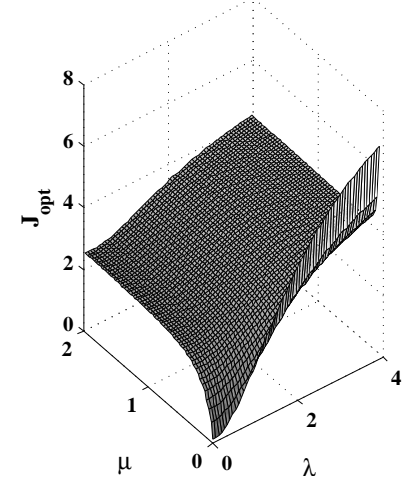

Fig. b: $\Delta \mathbf{J}$

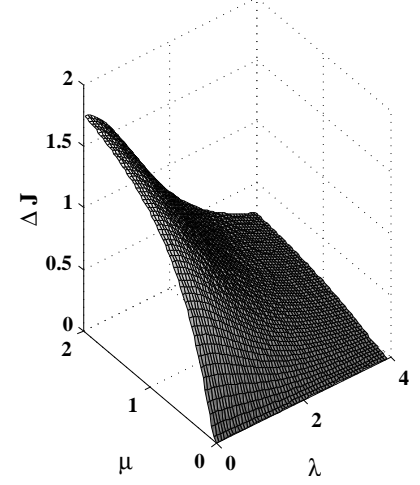

Fig. 2. Fig. a depicts the cost of using the optimal control (13). Fig. b illustrates the additional cost $\left(\Delta J=J_{\text {nonopt }}-J_{\text {opt }}\right)$ due to the control policy that is obtained by minimizing (2) and is optimal for every individual environment when there is no switching. This control results in a larger cost when the environmental switching rate is large, with respect to the protein degradation rate. The system starts from $x_{0}=0.9$ and in environment 1 with $\rho=0.1$ and $\lambda_{0}=\lambda_{1}=\lambda$.

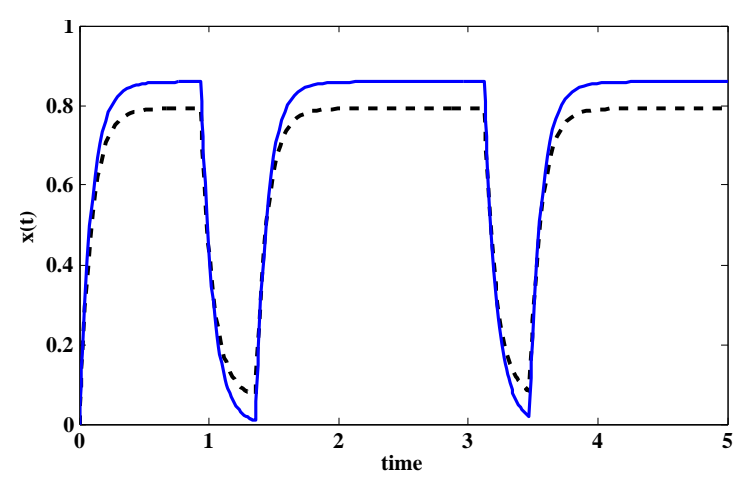

Fig. 3. Sample paths using the control strategies discussed in Section IV. The dashed line corresponds to the optimal controller in fluctuating environment while the solid line is the result of the controller which is optimal in each environment when there is no switching. The system starts from $x_{0}=0$ and in environment 1 with $\rho=0.1$ and $\lambda_{0}=\lambda_{1}=1, \mu=4$.

Figure 2 compares the cost of applying the control which is optimal in each environment (if there was no switching) and the optimal control policy (13) from Section II-B that takes into account that the environment changes stochastically. Figure 2.b illustrates that the optimal policy (13) results in a much smaller cost when the switching rate of the environment is large, when compared to the degradation rate of the protein. The biological implication of this observation is that an organism that evolved through natural selection in a variable environment is likely to exhibit specialization to the statistics that determine the changes in the environment. Opposite to what one could naively expect, such individual will typically not simply switch between responses that are optimal for the current environment, as if that environment were to remain static forever. Figure 3 illustrates sample paths of the system using the two control strategies discussed above. One can see that the controller that is optimal for the changing environment achieves a better cost by being conservative in its response to the environment.

\section{CONCLUSION}

We explored the effect of stochastically varying environments on the gene regulation problem. We used a mathematical model that combines stochastic changes in the environments with linear ordinary differential equations describing the concentration of gene product. Based on this model, we derived an optimal regulator that minimizes the infinite horizon discounted cost (6) with switching equilibria and showed that the regulator in each environment is an affine function of the expression level $\mathbf{x}$. We have also obtained a necessary and sufficient condition for the existence of an optimal control in terms of a set of LMI conditions. For future work, we plan to consider scenarios where the waiting times between the environmental changes follow arbitrary distributions. Furthermore, we plan to synthesize controllers that achieve a robust performance, where $H_{\infty}$ controllers might be applicable.

\section{REFERENCES}

[1] E. Dekel, S. Mangan and U. Alon, Environmental Selection of the Feed-Forward Loop Circuit in Gene Regulation Networks, Physical Biology, vol. 2, 2005, pp 1959-1972.

[2] R. Karmakar and I. Bose, Graded and Binary Responses in Stochastic Gene Expression, Physical Biology, vol. 1, 2004, pp 197-204.

[3] V. Shahrezaei and P.S. Swain, Analytical Distributions for Stochastic Gene Expression, Proceeding of National Academy of Science, vol. 105, 2008, pp 17256-17261.

[4] K. Yoshikawa, T. Tanaka, Y. Ida, C. Furusawa, T. Hirasawa and H. Shimizu, Comprehensive phenotypic analysis of single-gene deletion and overexpression strains of Saccharomyces cerevisiae, Yeast, vol. 28, 2011, pp 349-361.

[5] M.H. Davis, Markov Models and Optimization, Chapman \& Hall, London; 1993.

[6] J.P. Hespanha, A Model for Stochastic Hybrid Systems with Application to Communication Networks, Nonlinear Analysis, Special Issue on Hybrid Systems, vol. 62, 2005, pp 1353-1383.

[7] S. Zeiser, U. Franz, J. Mller and V. Liebscher, Hybrid Modeling of Noise Reduction by a Negatively Autoregulated System, Bulletin of Math Biology, vol. 71, 2009, pp 1006-1024.

[8] A. Singh and J.P. Hespanha, Stochastic Hybrid Systems for Studying Biochemical Processes, Phil. Trans. R. Soc. A, vol. 368, 2010, pp 4995-5011.

[9] E. Cinquemani, R. Porreca, G. Ferrari-Trecate and J. Lygeros, Subtilin Production by Bacillus Subtilis: Stochastic Hybrid Models and Parameter Identification, IEEE Transactions on Automatic Control, vol. 53, 2008, pp 38-50.

[10] E. Cinquemani, A. Milias, S. Summers and J. Lygeros, Stochastic Dynamics of Genetic Networks: Modelling and Parameter Identification, Bioinformatics, vol. 24, 2008, pp 2748-2754.

[11] M. Mariton, Jump Linear Systems in Automatic Control, Marcel Dekker, New York; 1990.

[12] Y. Ji and H.J. Chizeck, Controllability, Stabilizability, and ContinuousTime Markovian Jump Linear Quadratic Control, IEEE Transactions on Automatic Control, vol. 35, 1990, pp 777-788.

[13] Z. Gajic and I. Borno, Lyapunov Iterations for Optimal Control of Jump Linear Systems at Steady State, IEEE Transactions on Automatic Control, vol. 40, 1995, pp 1971-1975.

[14] M.W. Smiley and S.R. Proulx, Gene Expression Dynamics in Randomly Varying Environments, Journal of Mathematical Biology, vol. 61, 2010, pp 231-251.

[15] R.S. Abreu, L.O. Penalva, E.M. Marcotte and C. Vogel, Global Signatures of Protein and mRNA Expression levels, Molecular BioSystems, vol. 5, 2009, pp 1512-1526.

[16] U. Alon, An Introduction to Systems Biology: Design Principles of Biological Circuits, Chapman \& Hall, London; 2007.

[17] A. Wanger, Energy Constraints on the Evolution of Gene Expression, Molecular Biology and Evolution, vol. 22, 2005, pp 1365-1374. 
[18] C. Tanchot, F.A. Lemonnier, B. Perarnau, A.A. Freitas and B. Rocha, Differential requirements for survival and proliferation of CD8 naive or memory T cells, Science, vol. 276, 1997, pp 2057-2062.

[19] M.K. Slifka, R. Antia, J.K. Whitmire and R. Ahmed, Humoral immunity due to long-lived plasma cells, Immunity, vol. 8, 1998, pp 363-372.

[20] E. K. Deenick, A.V. Gett and P.D. Hodgkin, Stochastic model of T cell proliferation: a calculus revealing IL-2 regulation of precursor frequencies, cell cycle time, and survival, Immunol., vol. 170, 2003, pp 4963-4972.

[21] W. Rudin, Real and Complex Analysis, McGraw-Hill, New York; 19

[22] G.E. Dullerud and F. Paganini, A Course in Robust Control Theory, A Convex Approach, Springer, New York; 2000.

[23] T.Chiang and Y. Chow, On Eigenvalues and Annealing Rates, Mathematics of Operations Research, vol. 13, 1988, pp 508-511. 